\title{
A Superconnection for Riemannian Gravity as Spontaneously Broken SL $(4, R)$ Gauge Theory
}

\author{
Yuval Ne'eman \\ Sackler Faculty of Exact Sciencesף, Tel-Aviv University, Israel 69978 \\ and \\ Center for Particle Physics, University of Texas, Austin, Texas 78712
}

\begin{abstract}
A superconnection is a supermatrix whose even part contains the gaugepotential one-forms of a local gauge group, while the odd parts contain the (0-form) Higgs fields; the combined grading is thus odd everywhere. We demonstrate that the simple supergroup $\bar{P}(4, R)$ (rank=3) in Kac' classification (even subgroup $\overline{S L}(4, R)$ ) provides for the most economical spontaneous breaking of $\overline{S L}(4, R)$ as gauge group, leaving just local $\overline{S O}(1,3)$ unbroken. As a result, post-Riemannian SKY gravity yields Einstein's theory as a lowenergy (longer range) effective theory. The theory is renormalizable and may be unitary.
\end{abstract}

\section{Superconnections and the Electroweak $S U(2 / 1)$ as a model.}

The superconnection was introduced by Quillen [1] in Mathematics. It is a supermatrix, belonging to a given supergroup $S$, valued over elements belonging to a Grassmann algebra of forms. The even part of the supermatrix is valued over the gauge-potentials of the even subgroup $G \subset S$, (one-forms $B_{\mu}^{a} d x^{\mu}$ on the base manifold of the bundle, realizing the "gauging" of $G$ ). The odd part of the supermatrix, representing the quotient $S / G=H \subset S$, is valued over zero-forms in that Grassmann algebra, physically the Higgs multiplet $\Phi_{H}(x)$, in a spontaneously broken $G$ gauge theory; $\phi(x) \in \Phi_{H}(x)$, $<0|\phi(x)| 0>\neq 0$, thus leaving only a subgroup $F \subset G,[F, \phi]=0$ as the low-energy residual local symmetry. In quantum treatments which are set to reproduce geometrically the ghost fields and BRST equations [2], the Grassmann algebra is taken over the complete bundle variable. For simplicity in the presentation,we shall leave out that aspect in this work.

\footnotetext{
${ }^{1}$ Wolfson Distinguished Chair in Theoretical Physics
} 
The first physical example of a superconnection preceeded Quillen's theory. This was our $S U(2 / 1)$ (supergroup) proposal for an algebraically irreducible electroweak unification $[3,4]$. Lacking Quillen's generalized formulation, the model appeared to suffer from spin-statistics interpretative complications for the physical fields. The structural $Z_{2}$ grading of Lie superalgebras, as previously used in Physics (i.e. in supersymmetry) corresponds to the grading inherent in quantum statistics, i.e. to Bose/Fermi transitions, so that invariance under the supergroup represents symmetry between bosons and fermions. Here, however, though the superconnection itself does fit the quantum statistics ansatz, this is realized through the order of the forms in the Grassmann algebra, rather than through the quantum statistics of the particle Hilbert space! (both the $W_{\mu}^{ \pm}, Z_{\mu}^{0}, A_{\mu}$ on the one hand, and the Higgs field $\Phi_{H}$ on the other, are bosons). Moreover, the matter fields' (leptons or quarks) Hilbert space is $Z_{2}$-graded by fermion chiralities. The internal quantum numbers set by the $S U(2 / 1)$ assignments do display a perfect fit with the phenomenology (including the provision that integer charges - leptons - come in three states (e.g. $\left[\nu_{L}, e_{L} / e_{R}\right]$ ), whereas fractional charges quarks - come in four (e.g. $\left.\left[u_{R} / u_{L}, d L / d_{R}\right]\right)$, but they obviously do not lend themselves to a quantum statistics grading ansatz.

Two important sequels followed at that early stage. On the one hand, it was noted [5] that the method appears to apply to a large set of spontaneously broken symmetries, global or local. The example of the Goldstone-Nambu breaking of (global) chiral $S U(3) \otimes S U(3)$ was treated in detail in ref. [5], the relevant supergroup being the $Q(3)$ of Kac' classification [6] of the simple superalgebras. This is an exceptional supergroup we had encountered earlier [7], precisely because of its physical relevance. The second development was an improved understanding of both the physics and the mathematics of the juxtaposition of the two graded systems - on the one hand, the supergroup as represented by its supermatrices and on the other hand the Grassmann algebra over which it is valued [8]. Tied to the traditional Yang-Mills derivation, however, our Grassmann-even elements, in the group-odd sector of the superconnection, started with two-forms, thus missing the desirable zero-forms, which Quillen could freely postulatef Our final treatment, with S. Sternberg [9], availed itself of the advantage deriving from Quillen's generalized

\footnotetext{
${ }^{2}$ Our 1982 solution nevertheless did include a fitting scalar field, within the extended "ghost" system corresponding to the forms being taken over the entire bundle
} 
formalism, also applying the method to a further unification [10], including QCD and a $(2 k)$ generations structure, using $S U(5+k / 1)$.

One last 'technical' point relates to the multiplication of supermatrices. To stay within the axioms of matrix multiplication, terms in the product have to take appropriate signatures:

$$
\begin{aligned}
& {\left[\left(\begin{array}{cc}
A & B \\
C & D
\end{array}\right)\right] \cdot\left[\left(\begin{array}{cc}
A^{\prime} & B^{\prime} \\
C^{\prime} & D^{\prime}
\end{array}\right)\right]=} \\
& {\left[\left(\begin{array}{cc}
A \wedge A^{\prime}+(-1)^{b} B \wedge C^{\prime} & (-1)^{a} A \wedge B^{\prime}+B \wedge D^{\prime} \\
C \wedge A^{\prime}+(-1)^{d} D \wedge C^{\prime} & (-1)^{c} C \wedge B^{\prime}+D \wedge D^{\prime}
\end{array}\right)\right]}
\end{aligned}
$$

where $n=a, b, c, d$ are the respective orders of the n-forms $A, B, C, D$ in the Grassmann algebra.

The next installment came from Connes' noncommutative geometry (NCG), generalizing to discrete geometries some geometrical concepts (such as distances) till then defined only for continuous spaces. Connes and Lott [11] used the new formalism to reproduce the electroweak theory, providing it with a geometric derivation: the base manifold is $Z_{2} \otimes M^{3 / 1}=M_{L} \oplus M_{R}$, where $Z_{2}$ is a discrete space containing just two points $L, R$ representing chiralities and $M^{3 / 1}$ is Minkowski spacetime. NCG defines a space by the functions and Hilbert space states living on it and the operators acting on that Hilbert space. Here, parallel-transport within $M_{L}$ (or within $M_{R}$ ) is performed by $D=d+B(G)$, with $B$ standing for the relevant gauge potentials and $G=S U(2)_{W} \times U(1)_{Y}$. Moving, however, from a state sitting over a point in $M_{L}\left(\right.$ say $\left.\nu_{L}^{e}(x)\right)$ to one sitting over a point in $M_{R}\left(\right.$ say $\left.e_{R}(x)\right)$ requires a scalar "connection" $\Phi_{H}(x)$. In this case, its $G$ quantum numbers are entirely fixed by the matter fields' selected assignments; this includes the Lorentz scalar nature of $\Phi_{H}$, due to its having to relate e.g. $e_{L}^{-}$to $e_{R}^{-}$in a Yukawa term (the $\gamma^{0}$ is provided for by the Dirac operator, acting on the Hilbert space, in the definition of the noncommutative geometry).

The link with our $S U(2 / 1)$ superconnection was provided by Coquereaux, Scheck and coworkers of the "Marseilles-Mainz group" [12-16]. They found that by slightly modifying the Connes axioms, $\mathrm{su}(2 / 1)$ emerges naturally as the superalgebra of the form-calculus over the discrete $Z_{2}$ of the chiralities, while the superconnection for the product space $Z_{2} \otimes M^{3 / 1}$ is an $\mathrm{SU}(2 / 1)$

\footnotetext{
${ }^{3}$ The authors of ref. [11] worked on Euclidean $M^{4}$, instead of $M^{3 / 1}$ for technical reasons.
} 
group-element. This also finally exorcizes the apparent difficulty with the non-spin-statistics grading of the matter fields and explains how the grading can be related to chiralities instead. Moreover, the parallel-transport operator is found to require an additional "matrix derivative" $\delta_{H}$, relating "twin" states in $M_{L}$ and $M_{R}$, such as $e_{L}$ and $e_{R}$, etc.. (this is the role of $\beta$ in the Dirac $\gamma^{\mu}$ calculus). With this additional term, the curvature-squared Lagrangian $\hat{R} \wedge^{*} \hat{R}$ for $S=S U(2 / 1)$ contains the complete Weinberg-Salam Lagrangian. Indeed, $\hat{R}=R_{G}+(1 / 2)\left\{\Phi_{H}, \Phi_{H}\right\}+D_{G} \Phi_{H}+\delta_{H} \Phi_{H}$, with $R(G)=$ $d B+(1 / 2) B \wedge B$. In squaring, the second term in $\hat{R}$ provides for the $\lambda \Phi^{4}$ and the fourth provides for the negative mass-squared piece of the Higgs potential.

\section{Riemannian Gravity as deriving from a broken SKY Affine Gravity}

The interst in deriving Einstein's Riemannian theory through the spontaneous symmetry breakdown of a non-Riemannian theory stems from quantum considerations. First, the quantization of gravity implies spacetime quantization at Planck energies (where the Compton wavelength is also the Schwarzschild radius, $\left.(h / 2 \pi m c)=2 G m / c^{2}\right)$. This quantization, in itself, represents a departure from Riemannian geometry. Secondly, the addition in the Lagrangian of terms quadratic in the curvatures renders the theory finite (the new terms dominate at high-energy and are dimensionless in the action); however, it is nonunitary, due to the appearance of $p^{-4}$ propagators. These are present because of the Riemannian condition $D g_{\mu \nu}=0$, relating the connection $\Gamma$ to the metric $g_{\mu \nu}$ (the Christoffel formula). Thus $\Gamma \simeq \partial g$ and $R=d \Gamma+(1 / 2) \Gamma \Gamma \simeq(\partial)^{2} g+(\partial g)^{2}$ and $R^{2}$ will involve $p^{4}$ terms in momentum space and thus $p^{-4}$ propagators. These can then be rewritten as differences between two S-matrix poles - one of which is then a ghost, due to the wrong sign of its residue. It seems therefore worth trying to reconstruct gravity so that the Riemannian condition will only constrain the low-energy end of the theory, as an effective result in that regime. The high-energy theory, i.e. prior to symmetry breakdown, should have as its anholonomic (gauge) group the metalinear $\overline{S L}(4, R)$. . 7 We have investigated a model [17-20] based on either $\overline{S L}(4, R)$ or $\overline{G L}(4, R)$, containing the Stephenson-Kilmister-Yang

\footnotetext{
${ }^{4}$ The conformal $S U(2,2)=\overline{S O}(4,2)$ or the super-deSitter $O S p(4 / 1)$ of McDowellMansouri would still be Riemannian.
} 
(SKY) Lagrangian [21-23] plus a term linear in the curvature, and proved the Yang-Mills-like renormalizability and BRST invariance of the quantum Lagrangian. Whether the theory is unitary is not known at this stage, due to the presence of a $p^{-4}$ term as input in the gauge-fixing term of the quantum Lagrangian $^{[19-20]}$.

In such theories, (a) the $G=\overline{S L}(4, R)$-invariant $\hat{R}(G) \wedge{ }^{*} \hat{R}(G)$ SKY Lagrangian has to have its symmetry broken by a Higgs field corresponding to an $\overline{S L}(4, R)$ multiplet containing a Lorentz-scalar component, to ensure that $F=\overline{S O}(1,3)$. In the algebraic structure we use (the superalgebra of $S=\bar{P}(4, R))$, this includes a metric-like symmetric tensor $(a, b=0, . .3$ are anholonomic indices supporting the local action of $S$ and its subgroups) $\Phi_{\{a b\}}(x)$, and the Lorentz scalar is given by $\phi=\Phi_{a b} \eta^{a b}$, where $\eta_{a b}$ is either the trace (for Euclidean signature situations) or the Minkowski metric. Thus $<0|\phi(x)| 0>\neq 0$. (b) Those components of the connection $\Gamma_{b}^{a}(x)$ which serve to gauge $G / F=\overline{S L}(4, R) / \overline{S O}(1,3)$ should acquire masses in the spontaneous breakdown procedure. As in the electroweak case, we should have in the Higgs multiplet, components which - in the Unitary gauge - will have become the longitudinal (spin) components of the (now massive) $G / F$ elements of the connection. In our construction, these are precisely the 9 components of $\Phi_{\{a b\}}$, after removal of the trace (or Minkowski-trace). (c) Any remaining components of $\Phi_{H}(x)$ should acquire masses and exist as free particles. In the $\bar{P}(4, R)$ model, $\Phi_{H}(x)=\Phi_{\{a b\}}(x) \oplus \Phi_{[a b]}(x)$, i.e. there is, in addition, an antisymmetric field $\Phi_{[a b]}(x)$, which indeed acquires a Planck-scale mass.

\section{The simple superalgebra $p(4, R)$.}

The defining representation of the generating superalgebra of the $\bar{P}(4, R)$ supergroup is an $8 \times 8$ matrix, divided into quadrants. $I$ and $I V$ carry the $\operatorname{sl}(4, R)$ algebra, with $I$ in the covariant representation $\sum_{\tilde{b}}^{\tilde{a}}(a, b=0,1,2,3$ and the tildes indicate tracelessnes $\left.\operatorname{tr} \sum_{\tilde{b}}^{\tilde{a}}=0\right)$ and $I V$ in the contravariant, i.e. $I V=-I^{T}\left({ }^{T}\right.$ indicates transposition $)$. In the off-diagonal quadrants, $I I=\Sigma_{b\}}^{\{a}$ carries the 10 symmetric matrices of $g l(4, R)$ and $I I I=\Sigma_{b]}^{[a}$ carries its 6 antisymmetric matrices. There are thus altogether 31 generators, of which $15 Q_{\tilde{b}}^{\tilde{a}}$ are even, representing the action of $\operatorname{sl}(4, R)$ and $16 N_{b}^{a}$ are odd, of which 10 are the symmetric $N^{+}=T$, and 6 the antisymmetric $N^{-}=M$,exhausting the set of generators of $g l(4, R)$ (we use the notation of ref. [24], i.e. the T, $M$ are the shears and Lorentz generators, respectively). 
We shall also have occasion to use the nonsimple completion $\overline{g P}(4, R)$ in which the $\overline{S L}(4, R)$ even subgroup is completed to $\overline{G L}(4, R)$, without any change in the $\bar{P}(4, R)$ itself.

The simple superalgebra is thus given as,

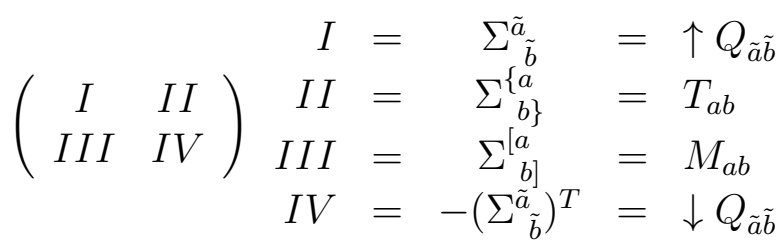

and

$$
\begin{aligned}
& Q_{a b}:=\left(\sum_{\tilde{\tilde{b}}}^{\tilde{a}}\right)_{I} \oplus-\left(\sum_{\tilde{b}}^{\tilde{a}}\right)_{I V}^{T} \\
& N_{a b}^{+}:=\left(\sum_{b\}}^{\{a}\right)_{I I} \\
& N_{a b}^{-}:=\left(\Sigma_{b]}^{[a}\right)_{I I I}
\end{aligned}
$$

To formulate the super-Lie bracket, we choose to replace the two-index (vector) notation by a single (matrix) index, as in $\mathrm{SU}(2)$ or $\mathrm{SU}(3)$ usage. We select an $\mathrm{SU}(4)$ basis $(4 \times 4$, " $\nu$ " matrices $)$ in which the $i=1 . .8$ correspond to setting the $\mathrm{SU}(3) \lambda_{i}$ matrices in the upper left-hand corner of the $\nu$ matrix with that index and define similar matrices for the rest. Since we are dealing with $S L(4, R)$ rather than $S U(4)$, we have to multiply the real matrices by $\sqrt{-1}$, thus making these generators noncompact. With $\sigma_{i}$ denoting the Pauli matrices, and $\left[\sigma_{i}\right]_{1,2}$ denoting a $\sigma_{1}$ matrix placed in the $[1,2]$ rows and 
columns of the $\nu$ matrix, we have a basis,

$$
\begin{aligned}
\nu_{1} & =i \lambda_{1}=i\left[\sigma_{1}\right]_{1,2} \\
\nu_{2} & =\lambda_{2}=\left[\sigma_{2}\right]_{1,2} \\
\nu_{3} & =i \lambda_{3}=i\left[\sigma_{3}\right]_{1,2} \\
\nu_{4} & =i \lambda_{4}=i\left[\sigma_{1}\right]_{1,3} \\
\nu_{5} & =\lambda_{5}=\left[\sigma_{2}\right]_{1,3} \\
\nu_{6} & =i \lambda_{6}=i\left[\sigma_{1}\right]_{2,3} \\
\nu_{7} & =\lambda_{7}=\left[\sigma_{2}\right]_{2,3} \\
\nu_{8} & =i \lambda_{8}=(i / \sqrt{3}) \operatorname{diag}(1,1,-2) \\
\nu_{9} & =--=i\left[\sigma_{1}\right]_{1,4} \\
\nu_{10} & =--=\left[\sigma_{2}\right]_{1,4} \\
\nu_{11} & =--=i\left[\sigma_{1}\right]_{2,4} \\
\nu_{12} & =--=\left[\sigma_{2}\right]_{2,4} \\
\nu_{13} & =--=i\left[\sigma_{1}\right]_{3,4} \\
\nu_{14} & =--=\left[\sigma_{2}\right]_{3,4} \\
\nu_{15} & =--=(i / \sqrt{6}) \operatorname{diag}(1,1,1,-3)
\end{aligned}
$$

Using the definition of the $f_{i j k}$ (totally antisymmetric) and $d_{i j k}$ (totally symmetric) coefficients of $s u(3)$, generalized to $s u(4)$ and corrected by the factors $\sqrt{-1}$ for the symmetric matrices in the $s u(4)$ basis when changing to $s l(4, R)$ as indicated above, we get coefficients $\hat{f}_{i j k}$ and $\hat{d}_{i j k}$ whose symmetry properties are thus reduced to the first two indices only. We can now write the Lie superbrackets as,

$$
\begin{aligned}
{\left[Q_{i}, Q_{j}\right] } & =2 i \hat{f}_{i j k} Q_{k} \\
{\left[Q_{i}^{A}, N_{j}^{+}\right] } & =2 i \hat{f}_{i j k} N_{k}^{+} \\
{\left[Q_{i}^{A}, N_{0}^{+}\right] } & =0 \\
{\left[Q_{i}^{T}, N_{j}^{+}\right] } & =2 \hat{d}_{i j k} N_{k}^{+} \\
{\left[Q_{i}^{T}, N_{0}^{+}\right] } & =2 i N_{i}^{+} \\
{\left[Q_{i}^{T}, N_{j}^{-}\right] } & =2 i \hat{d}_{i j k} N_{k}^{-} \\
{\left[Q_{i}^{A}, N_{j}^{-}\right] } & =0 \\
\left\{N_{i}^{+}, N_{j}^{-}\right\} & =2 \hat{d}_{i j k} Q_{k}^{A} \\
\left\{N_{0}^{+}, N_{i}^{-}\right\} & =2 i Q_{i}^{A}
\end{aligned}
$$




\section{The Superconnection, Supercurvature and the Lagrangian}

At this stage we set up the relevant superconnection à la Quillen, as an ad hoc algorithm (we shall later discuss the possibility of generating it from the matter fields' fiber bundle, by using a Connes-Lott type of product base space). The superconnection will thus be given as

$$
\left(\begin{array}{ccc}
\Gamma_{\tilde{a}}^{\tilde{b}} \Sigma_{\tilde{b}}^{\tilde{a}} & \Phi{ }^{\left.b_{a}\right\}} \Sigma^{\{a} \\
\Phi & {\left[b_{a]} \Sigma_{b]}^{[a}\right.} & -\Gamma_{\tilde{b}}^{\tilde{a}} \Sigma_{\tilde{a}}^{\tilde{b}}
\end{array}\right)
$$

The nonvanishing v.e.v. field $\phi(x)=\Phi_{0}^{+}$will occupy the main diagonal of quadrant II. This will also be the structure of the matrix derivative $\delta$,

$$
\delta=\left(\begin{array}{cc}
0 & i .1_{4 \times 4} \\
0 & 0
\end{array}\right)
$$

The resulting (generalized) curvature is then,

$$
\hat{R}=R(G)++\left\{\Phi_{+}, \Phi_{-}\right\}+D \Phi_{+}+D \Phi_{-}+\delta \Phi^{-}
$$

where $\Phi_{+}, \Phi_{-}$respectively denote the symmetric (in quadrant II) and antisymmetric (in quadrant III) components of $\Phi_{H}$. The first two terms arise for the $15 \hat{R}^{G}$, the last three appear for the $16 \hat{R}^{H}$. In addition to its action on the Grassmann algebra - replacing an n-form by a (4-n)-form - the * duality operator conjugates the supermatrix. The $\hat{R} \wedge^{*} \hat{R}$ gauge Lagrangian will thus consist of the following terms

(a) $R \wedge^{*} R$, the SKY Lagrangian $[21-23]$,

(b) $\left|\Phi^{-}\right|^{2} \phi^{2}$, the $\Phi^{-}$mass term, once $<0\left|\Phi_{0}^{+}\right| 0>\neq 0$.

(c) $\left|\left\{\Phi^{-}, \Phi^{+}\right\}\right|^{2}$, the quartic Higgs potential $V_{4}$.

(d) $\left(D \Phi_{+}\right)^{2}$ the $\Phi_{+}$kinetic energy and gauge interaction,

(e) $\left(D \Phi_{-}\right)^{2}$, the $\Phi_{-}$kinetic energy and gauge interaction,

(f) $\left|\delta \Phi^{-}\right|^{2}$, the "negative squared mass" term $V_{2}$, triggering the spontaneous breakdown of local $G$ symmetry, through $\frac{\partial\left(V_{2}+V_{4}\right)}{\partial\left|\Phi^{-}\right|^{2}}=0$

(g) There is no $\left\{\Phi^{+}, \Phi_{0}^{+}\right\}$term, so that the 9 traceless components of $\Phi^{+}$do not acquire mass. Moreover, they become the longitudinal $G / F=$ $S L(4, R) / S O(4)$-gauging components of the connection, which acquire mass under the spontaneous symmetry breakdown. 


\section{The matter Lagrangian and Connes-Lott like Geometry}

We now discuss a Connes-Lott like derivation. We stick to the chiral $Z_{2}$ grading, i.e. to the product space $Z_{2} \otimes M^{3,1}=M_{L} \oplus M_{R}$ as base space. The matter fields will consist of world spinor manifields [24-28], the (spinorial) infinite-component representations of the double-coverings $\overline{G L}(4, R)$ and $\overline{S L}(4, R)$, which, for several decades, were wrongly assumed not to exist - in the General Relativity literature - even though a well-known algebraic theorem states that the topology of a Lie group is that of its maximal compact subgroup,i.e. $S O(4) \subset S L(4, R)$ and accordingly $S U(2) \times S U(2) \subset \overline{S L}(4, R)$. We refer the reader to the relevant literature, e.g. Chapter 4 and Appendix C of ref. [28].

The appropriate choice is the manifield based on $\mathcal{D}\left(\frac{1}{2}, 0\right) \oplus \mathcal{D}\left(0, \frac{1}{2}\right)$, where $\mathcal{D}$ denotes the $\overline{S L}(4, R)$ irreducible representation (applying the deunitarizing automorphism $^{[24]} \mathcal{A}$ ) and $\left(\frac{1}{2}, 0\right),\left(0, \frac{1}{2}\right)$ denote the lowest representations of the $S O(4)$ subgroup, here a nonunitary representation of $S L(2, C)$, namely a Dirac spinor. We refer the reader to the literature - see figs. 3,4,5 of ref. [28] - for a detailed discussion of this field. Obviously, for a massless field, $\left(\frac{1}{2}, 0\right)$ and $\left(0, \frac{1}{2}\right)$ respectively form the fibres over $M_{L}$ and $M_{R}$, with $G=\overline{S L}(4, R)$ as a common structure group. The odd connection bridging parallel-transport between points on the bundles constructed over $M_{L}$ and $M_{R}$ has to contain (e.g. in a Yukawa-like term) a $\left(\frac{1}{2}, \frac{1}{2}\right) \gamma^{0}$-supported scalar $\Phi_{0}^{+}$at least. However, considering the structure of the manifield (see figs. 4,5 in ref. [28]), the $N^{+}$generators with their $(1,1)$ action and the $\Phi^{+}$ connections, are just what is needed to make a one-step bridging along the fibre. The role of the $\Phi^{-}$, however, is rather unclear, since the $N^{-}$generators, with their $(1,0) \oplus(0,1)$ action, act trivially. We have to be satisfied with the $N^{-}$emerging from the algebraic consistency of the supergroup structure, in going from the pure Connes-Lott to its Marseilles-Mainz modification.

\section{References}

[1] D. Quillen, Topology 24 (1985) 89.

[2] J. Thierry-Mieg, J. Math. Phys. 21 (1980) 2834. 
[3] Y. Ne'eman, Phys. Lett. B81 (1979) 190.

[4] D.B. Fairlie, Phys. Lett. B82 (1979) 97.

[5] Y. Ne'eman and J. Thierry-Mieg, Proc. Nat. Acad. Sci. USA 77 (1980) 720.

[6] V.G. Kac, Func. Analysis and Appl. 9 (1975) 91; also Comm. Math. Phys. 53 (1977) 31. See also V. Rittenberg, in Group Theoretical Methods in Physics (Proc. Tübingen 1977), P. Kramer and A. Rieckers eds., Springer Verlag Lecture NOtes in Physics 79 (Berlin, Heidelberg, New York 1977), pp. 3-21.

[7] L. Corwin, Y. Ne'eman and S. Sternberg, Rev. Mod. Phys. 47 (1975) 573.

[8] J. Thierry-Mieg and Y. Ne'eman, Proc. Nat. Acad. Sci. USA 79 (1982) 7068.

[9] Y. Ne'eman and S. Sternberg, Proc. Nat. Acad. Sci. USA 87 (1990) 7875 .

[10] Y. Ne'eman and S. Sternberg, Proc. Nat. Acad. Sci. USA 77 (1980) 3127.

[11] A. Connes and J. Lott, Nucl. Phys. (Proc. Suppl.) 18B (1990) 29.

[12] R. Coquereaux, R. Haussling, N.A. Papadopoulos and F. Scheck, Int. J. Mod. Phys. A7 (1992), 2809.

[13] R. Coquereaux, G. Esposito-Farese and F. Scheck, Int. J. Mod. Phys. A7 (1992) 6555.

[14] N.A. Papadopoulos, J. Plass and F. Scheck, Phys. Lett. B324 (1994) 380.

[15] N.A. Papadopoulos and J. Plass, Mainz preprint MZ-TH 95-11.

[16] F. Scheck, preprint hep-th/9701073 (Jan. 97).

[17] Y. Ne'eman and Dj. Šijački, Phys. Lett. B200 (1988) 489. 
[18] C.-Y. Lee and Y. Ne'eman, Phys. Lett. B233 (1989) 286.

[19] C.-Y. Lee and Y. Ne'eman, Phys. Lett. bf B242 (1990) 59.

[20] C.-Y. Lee, Class. Quantum Grav. 9 (1992) 2001.

[21] Stephenson, Nuovo Cimento 9 (1958) 263.

[22] C.W. Kilmister and D.J. Newman, Proc. Cam. Phil. Soc. 57 (1961) 851.

[23] C.N. Yang, Phys. Rev. Lett. 33 (1974) 445.

[24] Dj. Šijački and Y. Ne'eman, J. Math. Phys. 26 (1985) 2457.

[25] Y. Ne'eman, Proc. Nat. Acad. Sci. USA 74 (1977) 4157.

[26] Y. Ne'eman, Ann. Inst. H. Poincaré A28 (1978) 369.

[27] Y. Ne'eman and Dj. Šijački, Int. J. Mod. Phys. A2 (1987) 1655.

[28] F.W. Hehl, J.D. McCrea, E.W. Mielke and Y. Ne'eman, Phys. Rep. 258 (1995) n1,2. 